\title{
PM.

\section{MTHFR C677T and A1298C polymorphisms and risk of lung cancer: a comprehensive evaluation}

\author{
Y. Yang ${ }^{1 *}$, L.J. Yang ${ }^{2 *}$, M.Z. Deng ${ }^{1}$, Y.Y. Luo', S. Wu' ${ }^{1}$, L. Xiong'1 , D. Wang1, \\ Y. Liu ${ }^{1}$ and H. Liu ${ }^{1}$ \\ ${ }^{1}$ West China Hospital, Sichuan University, Chengdu, China \\ 2Department of Respiration, East Branch, Sichuan Provincial People's Hospital, \\ Sichuan Academy of Medical Science, Chengdu, China \\ *These authors contributed equally to this study. \\ Corresponding author: H. Liu \\ E-mail: liuhao6304@hotmail.com \\ Genet. Mol. Res. 15 (2): gmr.15027615 \\ Received September 10, 2015 \\ Accepted December 2, 2015 \\ Published April 4, 2016 \\ DOI http://dx.doi.org/10.4238/gmr.15027615
}

\begin{abstract}
Results from previous studies on the association between methylenetetrahydrofolate reductase (MTHFR) polymorphisms C677T and $\mathrm{A} 1298 \mathrm{C}$ and lung cancer have been conflicting. The aim of this metaanalysis was to clarify the effect of MTHFR polymorphisms on the risk of lung cancer. An electronic search of PubMed, EMBASE, the Cochrane library, and the China Knowledge Resource Integrated Database for papers on C677T and A1298C and susceptibility to lung cancer was performed. The STATA software (Version 13.0) was used for statistical analysis. Statistical heterogeneity, tests of publication bias, and a sensitivity analysis were performed. Twenty-six studies on C677T (12,324 cases and 12,532 controls) and thirteen studies on A1298C (6773 cases and 8207 controls) were included in the meta-analysis. The MTHFR C677T polymorphism showed significant pooled ORs for the homozygote comparison (TT versus CC: $\mathrm{OR}=1.518,95 \% \mathrm{Cl}=1.220-1.890)$, heterozygote comparison (CT versus CC: $\mathrm{OR}=1.053,95 \% \mathrm{Cl}=0.940-1.179)$, dominant model $(\mathrm{CT}+$
\end{abstract}


TT versus CC: $\mathrm{OR}=1.143,95 \% \mathrm{Cl}=1.013-1.291)$, recessive model $($ TT versus $C T+C C: O R=1.435,95 \% \mathrm{Cl}=1.190-1.730)$, and additive model ( $\mathrm{T}$ versus $\mathrm{C}$ : $\mathrm{OR}=1.176,95 \% \mathrm{Cl}=1.066-1.298)$. In summary, our metaanalysis showed that the MTHFR C677T polymorphism is associated with a significant increase in lung cancer risk in Asian and overall populations, but not in Caucasian populations. However, no significant association between the MTHFR A1298C polymorphism and lung cancer risk was found in either the Caucasian or Asian group with any genetic models.

Key words: MTHFR; C677T; A1298C; Polymorphisms; Lung cancer; Meta-analysis

\section{INTRODUCTION}

Approximately 14.1 million new cancer cases and 8.2 million deaths occurred in 2012 worldwide. Lung cancer is the leading cause of cancer death among males in both developed and developing countries, and has surpassed breast cancer as the leading cause of cancer death among females in developed countries (Torre et al., 2015). Lung cancer risk is not fully understood, but it has been commonly accepted to be related to many factors, including genetic and/or environmental factors. Among these, smoking is regarded as an important risk factor for lung cancer (relative risk $=10-30$ compared with nonsmokers). However, lung cancer occurs in less than $20 \%$ of people who smoke throughout their life, suggesting that genetic factors may play a very important role in the development of lung cancer (Shields, 2002).

Previous epidemiological studies have demonstrated that consumption of fruits and vegetables is associated with a decreased risk of cancer, including lung cancer. Numerous constituents of fruits and vegetables are thought to contribute to this protective effect. One is folate, often in the form of folic acid (Shen et al., 2003). The enzyme MTHFR plays a critical role in the folate metabolism pathway by regulating the intracellular folate pool for synthesis and methylation of DNA. The MTHFR gene is located on chromosome 1 at the end of the short arm (1p36.6) and is $2.2 \mathrm{~kb}$ in length with a total of 11 exons (Goyette et al., 1994).

Several single nucleotide polymorphisms (SNP) in the MTHFR gene have been characterized, including C677T and A1298C. The C-to-T transition at nucleotide 677 in exon 4 is a point mutation that results in an amino acid substitution of valine for alanine, which decreases the thermal stability and reduces the activity of MTHFR. A reduced level of MTHFR substrate could lead to uracil misincorporation into DNA, diminished DNA repair, and increased frequency of chromosomal breaks and damage (Krajinovic et al., 2004). The MTHFR polymorphism A1298C (rs1801131) in exon 7, resulting in the replacement of glutamate with alanine at amino acid 429 (E429A), may increase serum folate levels, possibly influencing cancer risk (Parle-McDermott et al., 2006).

A number of studies have investigated the association between the two common MTHFR polymorphisms and the risk of lung cancer. However, the results remain controversial and previous studies have generally been small in size. In 2008, a meta-analysis based on eight studies reported the absence of associations between MTHFR C677T and A1298C and lung cancer (Mao et al., 2008). In 2009, a meta-analysis based on ten studies concluded that a possible increased risk exists for subjects carrying the MTHFR 677 TT genotype, but not for MTHFR 1298 CC (Boccia et al., 2009). To clarify the effect of the MTHFR C677T and A1298C polymorphisms on the risk of lung cancer, we have performed a meta-analysis including twenty-six studies on C677T (12,324 cases and 12,532 controls) and thirteen studies on A1298C (6773 cases and 8207 controls). 


\section{MATERIAL AND METHODS}

\section{Search strategy}

This meta-analysis was performed according to the standard MOOSE guideline (Stroup et al., 2000). PubMed, EMBASE, the Cochrane library, and the China Knowledge Resource Integrated Database (until January 1, 2015) were searched using the search terms "(C677T or A1298C or MTHFR) and (polymorphism or variants) and (lung cancer)". Only studies published in English or Chinese were included. Related reference articles were also searched to identify other relevant publications. Unpublished data and further information were also obtained from the corresponding authors.

\section{Inclusion and exclusion criteria}

Potential studies were selected based on the following inclusion criteria: 1) MTHFR C677T or A1298C polymorphisms and lung cancer were assessed; 2) human case-control design; 3) frequency of the MTHFR C677T or A1298C polymorphism was reported as number of cancer cases and controls according to the three variant genotypes of either polymorphism; and 4) published in English or Chinese. The criteria for exclusion were as follows: 1) not related to the MTHFR C677T or A1298C polymorphisms and lung cancer; 2) not a primary case-control study; 3) no usable or sufficient genotype data reported; 4) studies whose allele frequency in the control population deviated from the Hardy-Weinberg equilibrium at $\mathrm{P} \leq 0.01$; 5 ) case reports, letter to Editor, book chapters, or reviews. The study inclusion and exclusion procedures are summarized in Figure 1.

We contacted the corresponding authors by e-mail in order to obtain the absolute number of homozygous and heterozygous individuals in case and control groups for those papers reporting only the allele frequencies. If more than one article was published by the same author using the same case series, we selected one paper and excluded all others.

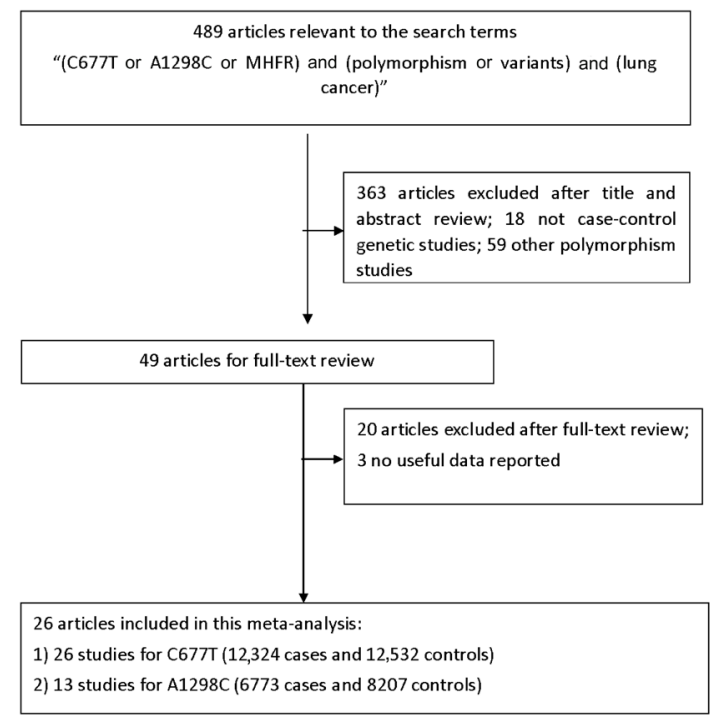

Figure 1. Inclusion and exclusion procedures. 


\section{Data extraction}

Two investigators independently extracted the data from all qualified studies using the selection standards listed above. Discrepancies were resolved through discussion until agreement was reached. We extracted the following information for each study: the first author's name, year of publication, the country in which the study was conducted, the source of the control group, evidence of Hardy-Weinberg equilibrium in controls, the sample size, the number of cases and controls with the CC/CT/TT or AA/AC/CC genotypes.

\section{Statistical analysis}

The STATA software (Version 13.0) was used for all statistical analyses. Two-sided P values $\leq 0.05$ were considered statistically significant. For the control groups for each study, the observed genotype frequencies of the MTHFR C677T and A1298C polymorphisms were evaluated for Hardy-Weinberg equilibrium. The strength of the association between the MTHFR C677T and A1298C polymorphisms and lung cancer risk was assessed by the odds ratios (ORs) with $95 \% \mathrm{Cls}$. The pooled ORs were calculated for the homozygote model, heterozygote model, dominant model, recessive model, and additive model. Cochran's Q-statistic and the $1^{2}$ metric were conducted to assess heterogeneity between studies, where $P<0.10$ and $I^{2}>50 \%$ were considered to indicate the existence of significant heterogeneity (Higgins and Thompson, 2002). If the heterogeneity test result returned $\mathrm{P}>0.1$, the pooled ORs were analyzed using the random-effect model; otherwise, the fixed-effect model was used. Sensitivity analyses were also performed after sequential removal of each study. Lastly, Begg's funnel plot and the Egger test were used to statistically examine publication biases.

\section{RESULTS}

\section{Characteristics of included studies}

Overall, twenty-six case-control studies with 12,324 lung cancer cases and 12,532 controls were retrieved based on the search criteria for lung cancer susceptibility related to the MTHFR C677T polymorphism. Thirteen studies also provide data on A1298C with 6773 lung cancer cases and 8207 controls. All studies included in the meta-analysis are summarized in Table 1 (Heijmans et al., 2003; Jeng et al., 2003; Siemianowicz et al., 2003; Shen et al., 2001, 2005; Shi et al., 2005; Zhang et al., 2005; Zou et al., 2006; Gemignani et al., 2007; Hung et al., 2007; Jing et al., 2007; Suzuki et al., 2007; Liu et al., 2008, 2009; Yang et al., 2010; Yao et al., 2010; Arslan et al., 2011; Cui et al., 2011a,b; Kiyohara et al., 2011; Cheng et al., 2011, 2012; Ma et al., 2012; Cai et al., 2014; Cavic et al., 2014; Yilmaz et al., 2014). No overlap occurred between the studies based on case or control participation. The genotype distributions in the controls for all studies were consistent with the Hardy-Weinberg equilibrium (Norton and Neel, 1965), with the exception of three studies.

\section{Results of the overall meta-analysis}

Our main results on the association between the MTHFR C677T and A1298C 
polymorphisms and lung cancer are listed in Table 2. The MTHFR C677T polymorphism showed significant pooled ORs for the homozygote comparison (TT versus CC: OR $=1.518,95 \% \mathrm{Cl}=$ 1.220-1.890), heterozygote comparison (CT versus CC: $\mathrm{OR}=1.053,95 \% \mathrm{Cl}=0.940-1.179$ ), dominant model $(C T+$ TT versus CC: $\mathrm{OR}=1.143,95 \% \mathrm{Cl}=1.013-1.291)$, recessive model ( $\mathrm{TT}$ versus $\mathrm{CT}+\mathrm{CC}$ : $\mathrm{OR}=1.435,95 \% \mathrm{Cl}=1.190-1.730$ ), and additive model $(\mathrm{T}$ versus $\mathrm{C}$ : $\mathrm{OR}=1.176,95 \% \mathrm{Cl}=1.066-1.298$ ). The MTHFR A1298C polymorphism showed non-significant pooled ORs for the homozygote comparison (CC versus AA: $\mathrm{OR}=1.073,95 \% \mathrm{Cl}=0.943-1.221$ ), heterozygote comparison (AC versus AA: $\mathrm{OR}=0.992,95 \% \mathrm{Cl}=0.925-1.064)$, dominant model $(A C+C C$ versus $A A: O R=1.004,95 \% C l=0.940-1.074)$, recessive model $(C C$ versus $A C+$ AA: $O R=1.073,95 \% \mathrm{Cl}=0.948-1.214)$, and additive model $(C$ versus $A: O R=1.015,95 \% \mathrm{Cl}$ $=0.964-1.070)$. We found no association between the MTHFR A1298C polymorphism and lung cancer risk, whereas a significant correlation existed between the MTHFR C677T polymorphism and lung cancer risk (Figure 2).

\begin{tabular}{|c|c|c|c|c|c|c|c|c|c|c|c|c|}
\hline Author & Year & Region & Ethnicity & & & Case & & & & Control & & Hardy-Weinberg $(\mathrm{P})$ \\
\hline MTHFR C677T & & & & Total & CC & CT & $\pi$ & Total & $\mathrm{CC}$ & CT & TT & \\
\hline Shen et al., 2001 & 2001 & USA & \begin{tabular}{|l} 
Caucasian \\
\end{tabular} & 550 & 241 & 252 & 57 & 554 & 245 & 252 & 57 & 0.508 \\
\hline Jeng et al., 2003 & 2003 & China & Asian & 59 & 36 & 22 & 1 & 232 & 123 & 95 & 14 & 0.438 \\
\hline Siemianowicz et al., 2003 & 2003 & \begin{tabular}{|l|} 
Poland \\
\end{tabular} & \begin{tabular}{|l|} 
Caucasian \\
\end{tabular} & 146 & 38 & 60 & 48 & 44 & 18 & 20 & 6 & 0.906 \\
\hline Heijmans et al., 2003 & 2003 & Netherlands & Caucasian & 44 & 23 & 17 & 4 & 793 & 399 & 329 & 65 & 0.806 \\
\hline Shi et al., 2005 & 2005 & USA & Caucasian & 1051 & 483 & 468 & 100 & 1141 & 498 & 519 & 124 & 0.516 \\
\hline Zhang et al., 2005 & 2005 & China & Asian & 505 & 120 & 230 & 155 & 500 & 160 & 231 & 109 & 0.138 \\
\hline Shen et al., 2005 & 2005 & China & Asian & 116 & 33 & 65 & 18 & 111 & 53 & 42 & 16 & 0.117 \\
\hline Zou et al., 2006 & 2006 & China & Asian & 100 & 24 & 52 & 24 & 100 & 39 & 48 & 13 & 0.767 \\
\hline Jing et al., 2007 & 2007 & China & Asian & 100 & 24 & 52 & 24 & 100 & 39 & 48 & 13 & 0.767 \\
\hline Suzuki et al., 2007 & 2007 & \begin{tabular}{|l|l|} 
Japan \\
\end{tabular} & Asian & 515 & 182 & 256 & 77 & 1030 & 379 & 474 & 177 & 0.170 \\
\hline Gemignani et al., 2007 & 2007 & \begin{tabular}{|l|} 
European \\
\end{tabular} & \begin{tabular}{|l} 
Caucasian \\
\end{tabular} & 247 & 104 & 107 & 36 & 259 & 131 & 103 & 25 & 0.473 \\
\hline Hung et al., 2007 & 2007 & \begin{tabular}{|l} 
France \\
\end{tabular} & Caucasian & 2169 & 1009 & 929 & 231 & 2803 & 1397 & 1147 & 259 & 0.288 \\
\hline Liu et al., 2008 & 2008 & China & Asian & 500 & 157 & 245 & 98 & 424 & 149 & 265 & 10 & $\mathrm{P}<0.05$ \\
\hline Liu et al., 2009 & 2009 & Taiwan & Asian & 358 & 205 & 124 & 29 & 716 & 362 & 291 & 63 & 0.679 \\
\hline Yang et al., 2010 & 2010 & China & Asian & 120 & 49 & 52 & 19 & 165 & 62 & 75 & 28 & 0.516 \\
\hline Yao et al., 2010 & 2010 & China & Asian & 93 & 27 & 46 & 20 & 106 & 36 & 51 & 19 & 0.899 \\
\hline Kiyohara et al., 2011 & 2011 & \begin{tabular}{|l|} 
Japan \\
\end{tabular} & Asian & 462 & 153 & 201 & 108 & 379 & $\begin{array}{ll}158 \\
\end{array}$ & 170 & 51 & 0.624 \\
\hline Arslan et al., 2011 & 2011 & Turkey & \begin{tabular}{|l|} 
Caucasian \\
\end{tabular} & 64 & 30 & 27 & 7 & 61 & 29 & 29 & 3 & 0.206 \\
\hline Cui et al., 2011b & 2011 & China & Asian & 438 & 58 & 240 & 140 & 641 & 121 & 325 & 195 & 0.483 \\
\hline Cheng et al., 2011 & 2011 & China & Asian & 178 & 49 & 58 & 71 & 180 & 47 & 88 & 45 & 0.767 \\
\hline Cui et al., 2011a & 2011 & Korean & Asian & 3938 & 1361 & 1909 & 668 & 1700 & 540 & 862 & 298 & 0.148 \\
\hline Cheng et al., 2012 & 2012 & China & Asian & 94 & 26 & 33 & 35 & 78 & 21 & 39 & 18 & 0.990 \\
\hline Ma et al., 2012 & 2012 & China & Asian & 120 & 20 & 54 & 46 & 60 & 22 & 28 & 10 & 0.830 \\
\hline Cavic et al., 2014 & 2014 & Serbia & \begin{tabular}{|l} 
Caucasian \\
\end{tabular} & 55 & 34 & 18 & 3 & 53 & 13 & 33 & 7 & 0.057 \\
\hline Yilmaz et al., 2014 & 2014 & Turkey & \begin{tabular}{|l} 
Caucasian \\
\end{tabular} & 100 & 55 & 38 & 7 & 100 & 51 & 43 & 6 & 0.433 \\
\hline Cai et al., 2014 & 2014 & China & Asian & 202 & 54 & 102 & 46 & 202 & 69 & 112 & 21 & $\mathrm{P}<0.05$ \\
\hline MTHFRA1298C & & & & Total & AA & $\mathrm{AC}$ & $\mathrm{CC}$ & Total & AA & $\mathrm{AC}$ & $\mathrm{CC}$ & \\
\hline Shen et al., 2001 & 2001 & USA & \begin{tabular}{|l} 
Caucasian \\
\end{tabular} & 550 & 261 & 246 & 43 & 554 & 265 & 249 & 40 & 0.072 \\
\hline Siemianowicz et al., 2003 & 2003 & Poland & \begin{tabular}{|l} 
Caucasian \\
\end{tabular} & 146 & 32 & 76 & 38 & 44 & 12 & 24 & 8 & 0.507 \\
\hline Shi et al., 2005 & 2005 & USA & \begin{tabular}{|l} 
Caucasian \\
\end{tabular} & 1051 & 480 & 462 & 109 & 1141 & 554 & 496 & 91 & 0.168 \\
\hline Zhang et al., 2005 & 2005 & China & Asian & 505 & 355 & 141 & 9 & 500 & 345 & 150 & 5 & $P<0.05$ \\
\hline Shen et al., 2005 & 2005 & China & Asian & 114 & 71 & 41 & 2 & 109 & 69 & 34 & 6 & 0.509 \\
\hline Jing et al., 2007 & 2007 & China & Asian & 100 & 70 & 28 & 2 & 100 & 68 & 30 & 2 & 0.528 \\
\hline Suzuki et al., 2007 & 2007 & \begin{tabular}{|l|l|} 
Japan \\
\end{tabular} & Asian & 512 & 341 & 149 & 22 & 1019 & 652 & 322 & 45 & 0.515 \\
\hline Hung et al., 2007 & 2007 & France & Caucasian & 2209 & 1031 & 960 & 218 & 2865 & 1285 & 1268 & 312 & 0.976 \\
\hline Liu et al., 2008 & 2008 & China & Asian & 500 & 341 & 141 & 18 & 517 & 364 & 142 & 11 & 0.509 \\
\hline Liu et al., 2009 & 2009 & Taiwan & Asian & 358 & 228 & 115 & 15 & 716 & 467 & 226 & 23 & 0.491 \\
\hline Kiyohara et al., 2011 & 2011 & \begin{tabular}{|l|l|} 
Japan \\
\end{tabular} & Asian & 462 & 278 & 154 & 30 & 379 & 239 & 122 & 18 & 0.633 \\
\hline Arslan et al., 2011 & 2011 & Turkey & Caucasian & 64 & 29 & 29 & 6 & 61 & 28 & 29 & 4 & 0.543 \\
\hline Cai et al., 2014 & 2014 & China & Asian & 202 & 55 & 106 & 41 & 202 & 65 & 102 & 35 & 0.642 \\
\hline
\end{tabular}


Table 2. Results of the overall meta-analysis.

\begin{tabular}{|c|c|c|c|}
\hline MTHFR C677T & & & \\
\hline Contrast & OR, $95 \% \mathrm{Cl}$ & Heterogeneity & $Z$ and $P$ \\
\hline TT versus CC & $1.518,[1.220,1.890]$ & chi-squared $=120.84($ d.f. $=25) P<0.001$, l-squared $=79.3 \%$ & $z=3.74 P=0.000$ \\
\hline CT versus $\mathrm{CC}$ & $1.053,[0.940,1.179]$ & chi-squared $=65.32$ (d.f. $=25) \mathrm{P}<0.001$, I-squared $=61.7 \%$ & $z=0.89 P=0.372$ \\
\hline $\mathrm{CT}+\mathrm{TT}$ versus $\mathrm{CC}$ & $1.143,[1.013,1.291]$ & chi-squared $=86.41$ (d.f. $=25) \mathrm{P}<0.001, \mathrm{I}$-squared $=71.1 \%$ & $z=2.16 P=0.031$ \\
\hline TT versus $\mathrm{CT}+\mathrm{CC}$ & $1.435,[1.190,1.730]$ & chi-squared $=108.37($ d.f. $=25) P<0.001$, l-squared $=76.9 \%$ & $z=3.78 P=0.000$ \\
\hline T versus $\mathrm{C}$ & $1.176,[1.066,1.298]$ & chi-squared $=120.43($ d.f. $=25) \mathrm{P}<0.001, \mathrm{l}$-squared $=79.2 \%$ & $z=3.23 P=0.001$ \\
\hline MTHFR A1298C & & & \\
\hline Contrast & $\mathrm{OR}, 95 \% \mathrm{Cl}$ & Heterogeneity & $\mathrm{Z}$ and $\mathrm{P}$ \\
\hline CC versus AA & $1.073,[0.943,1.221]$ & chi-squared $=15.07($ d.f. $=12) P=0.238$, I-squared $=20.4 \%$ & $z=1.07 P=0.283$ \\
\hline$A C$ versus $A A$ & $0.992,[0.925,1.064]$ & chi-squared $=4.96($ d.f. $=12) \mathrm{P}=0.959, \mathrm{I}$-squared $=0.0 \%$ & $z=0.22 P=0.829$ \\
\hline$A C+C C$ versus $A A$ & $1.004,[0.940,1.074]$ & chi-squared $=8.12($ d.f. $=12) P=0.775$, l-squared $=0.0 \%$ & $z=0.13 P=0.898$ \\
\hline$C C$ versus $A C+A A$ & $1.073,[0.948,1.214]$ & chi-squared $=13.01$ (d.f. $=12$ ) $P=0.368$, l-squared $=7.7 \%$ & $z=1.12 P=0.263$ \\
\hline$C$ versus $A$ & $1.015,[0.964,1.070]$ & chi-squared $=12.17($ d.f. $=12) P=0.432$, l-squared $=1.4 \%$ & $z=0.57 P=0.565$ \\
\hline
\end{tabular}
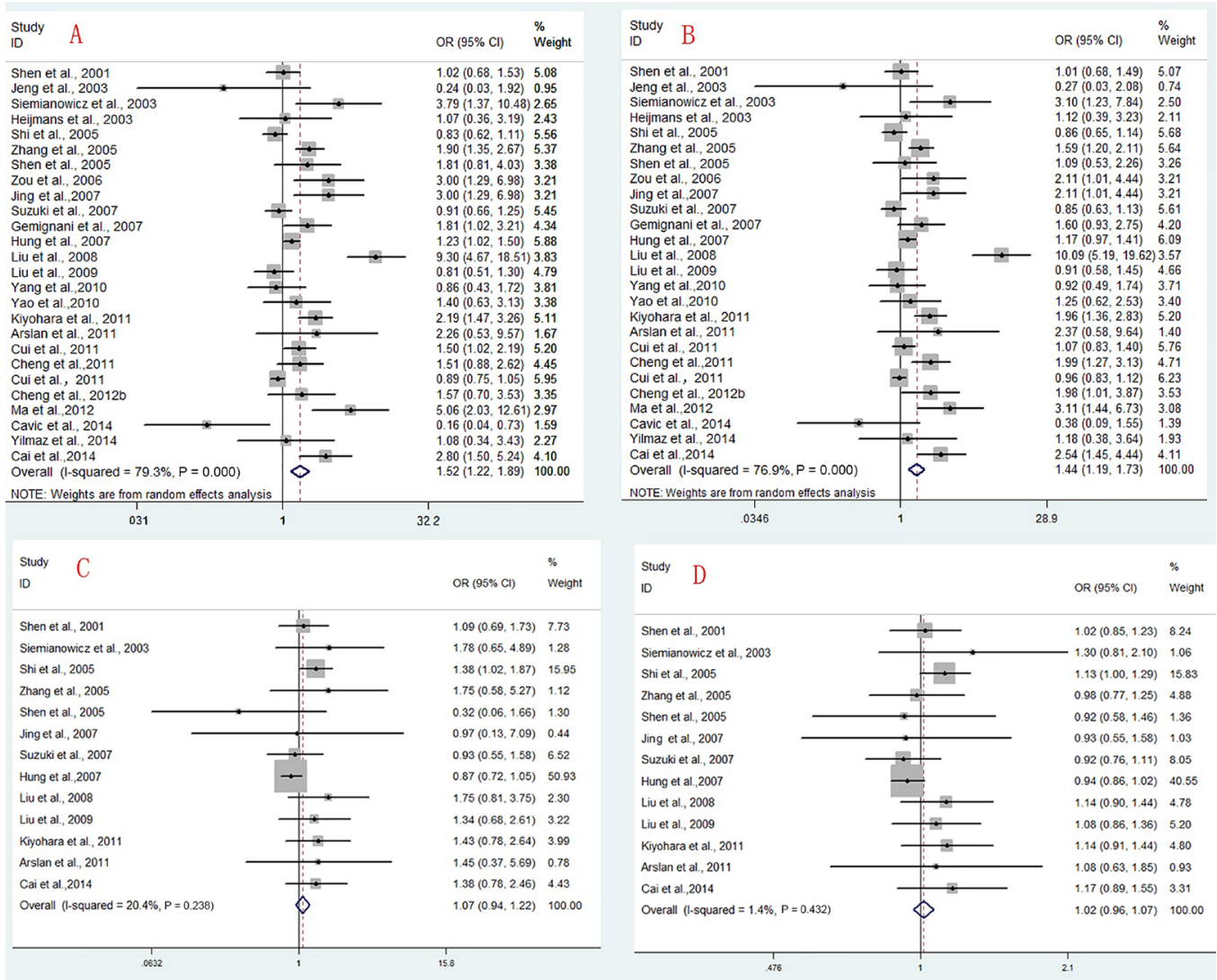

Figure 2. Overall meta-analysis for C677T (A, TT vs CC; B, TT vs CT + CC) and for A1298C (C, CC vs AA; D, C vs A) polymorphisms. 


\section{Subgroup analysis}

We performed a subgroup analysis stratified by ethnicity. We found that the MTHFR C677T polymorphism was associated with a significantly increased lung cancer risk in the Asian and overall populations. In the homozygote comparison (TT versus CC), the pooled OR was 1.722 $(95 \% \mathrm{Cl}=1.271-2.334, \mathrm{P}<0.001)$ for the Asian population. Under the recessive model (TT versus $\mathrm{CT}+\mathrm{CC})$, the pooled $\mathrm{OR}$ was $1.572(95 \% \mathrm{Cl}=1.215-2.033, \mathrm{P}=0.001)$ for the Asian population. However, we did not find an association between the A1298C polymorphism and lung cancer risk in the Asian group under any genetic model. Similarly, we found no significant association between either the C677T or A1298C polymorphism and lung cancer risk in the Caucasian group under any genetic model. The meta-analysis results for all genetic models are listed in detail in Table 3.

Table 3. Subgroup analysis stratified by ethnicity.

\begin{tabular}{|c|c|c|c|c|c|c|}
\hline \multicolumn{7}{|c|}{ MTHFR C677T } \\
\hline Ethnicity & Comparisons & $\begin{array}{c}\text { TT versus CC } \\
(\mathrm{OR}, 95 \% \mathrm{Cl}, \mathrm{Z}, \mathrm{P})\end{array}$ & $\begin{array}{c}\mathrm{CT} \text { versus } \mathrm{CC} \\
(\mathrm{OR}, 95 \% \mathrm{Cl}, \mathrm{Z}, \mathrm{P})\end{array}$ & $\begin{array}{l}\mathrm{CT}+\mathrm{TT} \text { versus } \mathrm{CC} \\
(\mathrm{OR}, 95 \% \mathrm{Cl}, \mathrm{Z}, \mathrm{P})\end{array}$ & $\begin{array}{l}\text { TT versus } \mathrm{CT}+\mathrm{CC} \\
(\mathrm{OR}, 95 \% \mathrm{Cl}, \mathrm{Z}, \mathrm{P})\end{array}$ & $\begin{array}{c}\text { T versus } \mathrm{C} \\
(\mathrm{OR}, 95 \% \mathrm{Cl}, \mathrm{Z}, \mathrm{P})\end{array}$ \\
\hline Caucasian & 9 & $\begin{array}{c}1.170,[0.859,1.593] \\
z=1.00 P=0.319\end{array}$ & $\begin{array}{c}0.978,[0.815,1.174] \\
z=0.24 P=0.813\end{array}$ & $\begin{array}{c}1.005,[0.822,1.229] \\
z=0.05 P=0.960\end{array}$ & $\begin{array}{c}1.146,[0.910,1.444] \\
z=1.16 P=0.247\end{array}$ & $\begin{array}{c}1.043,[0.888,1.224] \\
z=0.51 P=0.610\end{array}$ \\
\hline Asian & 17 & $\begin{array}{c}1.722,[1.271,2.334] \\
z=3.51 P<0.001\end{array}$ & $\begin{array}{c}1.107,[0.948,1.292] \\
z=1.28 P=0.201\end{array}$ & $\begin{array}{c}1.235,[1.046,1.458] \\
z=2.49 P=0.013\end{array}$ & $\begin{array}{c}1.572,[1.215,2.033] \\
z=3.45 P=0.001\end{array}$ & $\begin{array}{c}1.251,[1.096,1.429] \\
z=3.31 P=0.001\end{array}$ \\
\hline Overall & 26 & $\begin{array}{c}1.518,[1.220,1.890] \\
z=3.74 P<0.001\end{array}$ & $\begin{array}{c}1.053,[0.940,1.179] \\
z=0.89 P=0.372\end{array}$ & $\begin{array}{c}1.143,[1.013,1.291] \\
\mathrm{z}=2.16 \mathrm{P}=0.031\end{array}$ & $\begin{array}{c}1.435,[1.190,1.730] \\
z=3.78 P<0.001\end{array}$ & $\begin{array}{c}1.176,[1.066,1.298] \\
z=3.23 P=0.001\end{array}$ \\
\hline \multicolumn{7}{|c|}{ MTHFR A1298C } \\
\hline Ethnicity & Comparisons & $\begin{array}{c}\text { CC versus AA } \\
(\mathrm{OR}, 95 \% \mathrm{Cl}, \mathrm{Z}, \mathrm{P})\end{array}$ & $\begin{array}{c}\text { AC versus AA } \\
(\mathrm{OR}, 95 \% \mathrm{Cl}, \mathrm{Z}, \mathrm{P})\end{array}$ & $\begin{array}{l}\mathrm{AC}+\mathrm{CC} \text { versus } \mathrm{AA} \\
(\mathrm{OR}, 95 \% \mathrm{Cl}, \mathrm{Z}, \mathrm{P})\end{array}$ & $\begin{array}{l}\text { CC versus } \mathrm{AC}+\mathrm{AA} \\
(\mathrm{OR}, 95 \% \mathrm{Cl}, \mathrm{Z}, \mathrm{P})\end{array}$ & $\begin{array}{c}\text { C versus A } \\
(\mathrm{OR}, 95 \% \mathrm{Cl}, \mathrm{Z}, \mathrm{P})\end{array}$ \\
\hline Caucasian & 5 & $\begin{array}{c}1.021,[0.879,1.185] \\
z=0.27 P=0.789\end{array}$ & $\begin{array}{c}0.987,[0.903,1.079] \\
z=0.29 P=0.774\end{array}$ & $\begin{array}{c}0.993,[0.912,1.081] \\
\mathrm{z}=0.17 \mathrm{P}=0.865\end{array}$ & $\begin{array}{c}1.030,[0.893,1.187] \\
z=0.40 P=0.686\end{array}$ & $\begin{array}{c}1.002,[0.939,1.068] \\
z=0.05 P=0.957\end{array}$ \\
\hline Asian & 8 & $\begin{array}{c}1.247,[0.964,1.612] \\
z=1.68 P=0.092\end{array}$ & $\begin{array}{c}1.001,[0.895,1.119] \\
z=0.01 P=0.990\end{array}$ & $\begin{array}{c}1.023,[0.919,1.139] \\
z=0.42 P=0.674\end{array}$ & $\begin{array}{c}1.215,[0.949,1.556] \\
z=1.54 P=0.123\end{array}$ & $\begin{array}{c}1.043,[0.953,1.141] \\
z=0.91 P=0.361\end{array}$ \\
\hline Overall & 13 & $\begin{array}{c}1.073,[0.943,1.221] \\
z=1.07 P=0.283\end{array}$ & $\begin{array}{c}0.992,[0.925,1.064] \\
z=0.22 P=0.829\end{array}$ & $\begin{array}{c}1.004,[0.940,1.074] \\
z=0.13 P=0.898\end{array}$ & $\begin{array}{c}1.073,[0.948,1.214] \\
z=1.12 P=0.263\end{array}$ & $\begin{array}{c}1.015,[0.964,1.070] \\
z=0.57 P=0.565\end{array}$ \\
\hline
\end{tabular}

Ideally, we should perform a subgroup analysis stratified by type of lung cancer. However, we were unable to do this, as most of the studies included mixed types of lung cancer among the cases. Similarly, a subgroup analysis stratified by gender is also desirable, as gender may play a role in the development of lung cancer. However, only a few studies reported the absolute number of homozygous and heterozygous individuals in case and control groups stratified by gender.

\section{Test for heterogeneity}

For the C677T polymorphism, there was significant heterogeneity for the homozygote comparison (TT versus CC, $\chi^{2}=120.84$, d.f. $=25, \mathrm{P}<0.001, \mathrm{I}^{2}=79.3 \%$ ) and for the recessive model (TT versus CT + CC, $\chi^{2}=108.37$, d.f. $=25, P<0.001, \mathrm{I}^{2}=76.9 \%$ ). We assessed the source of the heterogeneity by region, publication year, control source, and sample size. However, we did not observe any sources that contributed to the substantial heterogeneity.

For the A1298C polymorphism, there was no significant heterogeneity for either the homozygote comparison ( $C C$ versus $A A, \chi^{2}=15.07$, d.f. $=12, P=0.238, \mathrm{I}^{2}=20.4 \%$ ) or the recessive model $\left(C C\right.$ versus $A C+A A, \chi^{2}=13.01$, d.f. $\left.=12, P=0.368, I^{2}=7.7 \%\right)$.

\section{Sensitivity analysis}

We conducted sensitivity analyses to ascertain the primary origin of the heterogeneity. The sensitivity analysis showed that no individual study had a marked effect on the pooled ORs (Figure $3 \mathrm{~A}$ and $\mathrm{B}$ ). 

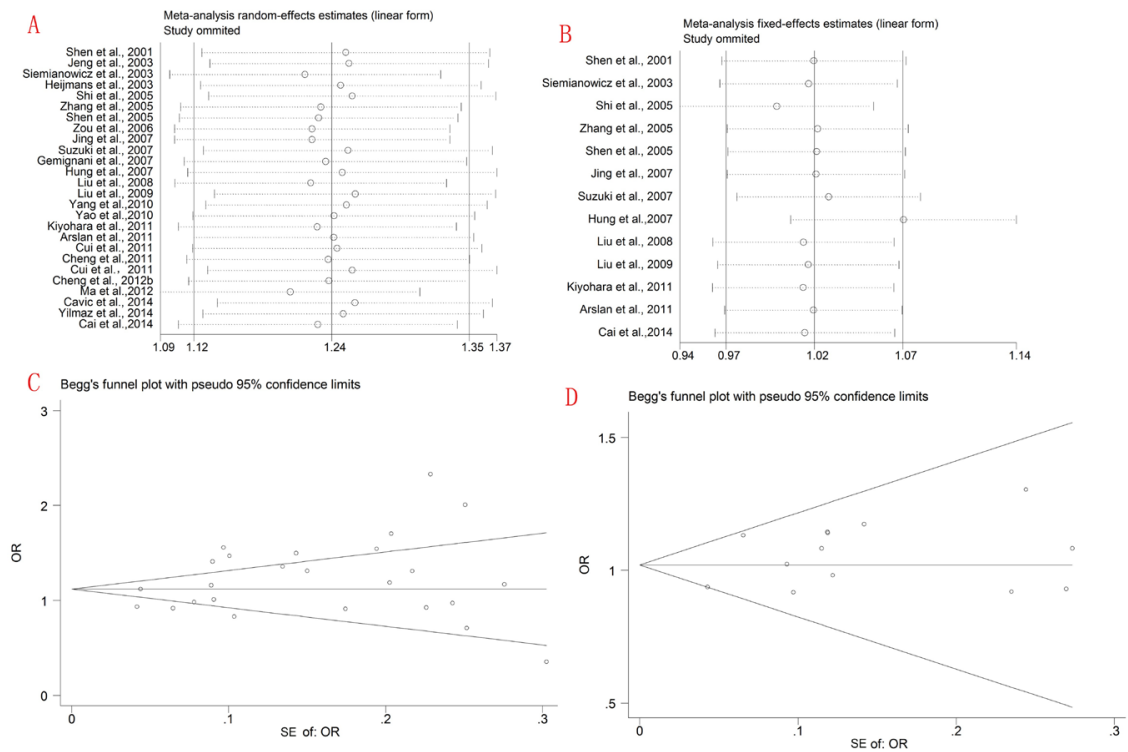

Figure 3. Sensitivity analysis and tests for publication bias.

\section{Publication bias}

A funnel plot was generated to assess publication bias (Figure $3 \mathrm{C}$ and D). Begg and Egger tests were performed to evaluate the funnel plot's symmetry statistically. The results showed no publication bias (Begg test $\operatorname{Pr}>|z|=0.440$ for C677T and $\operatorname{Pr}>|z|=0.669$ for A1298C).

\section{DISCUSSION}

Lung cancer is the leading cause of cancer death among males in both developed and developing countries and has surpassed breast cancer as the leading cause of cancer death among females in developed countries. Despite rapid advances in treatment over recent decades, the prognosis has not greatly improved: the 5-year survival rates for surgically resectable non-small cell lung cancer are still unsatisfactory and range from $19 \%$ for stage IIIA to $63 \%$ for stage IA (van Rens et al., 2000). Efforts toward primary prevention in addition to early detection have become more important. Lung cancer risk is commonly accepted to be multi-factorial, with genetic and/or environmental contributors. Although it is well known that cigarette smoking is a major cause of lung cancer, only $10-20 \%$ of lifetime smokers are known to develop lung cancer.

Folate is one of the micronutrients that provides protection against lung carcinogenesis (Voorrips et al., 2000). Shen et al. (2001) first examined the association between MTHFR gene polymorphisms and the risk of lung cancer. Subsequent case-control studies have provided controversial results. Small sample sizes, various ethnic groups, diets, environments, and methodologies may be responsible for the discrepancies. Two meta-analyses have been published, and neither detected any significant associations (Mao et al., 2008; Boccia et al., 2009). To clarify the effect of MTHFR polymorphisms C677T and A1298C on the risk of lung cancer, we performed a meta-analysis of twenty-six studies concerning C677T (12,324 cases and 12,532 controls) and thirteen studies concerning A1298C (6773 cases and 8207 controls). Our results 
showed no association between the MTHFR A $1298 \mathrm{C}$ polymorphism and lung cancer risk, whereas a significant correlation between the MTHFR C677T polymorphism and lung cancer risk existed in the overall population. In addition, we found that the C677T polymorphism was associated with a significantly increased lung cancer risk in Asian populations, but not in Caucasian populations. However, no significant association between the MTHFR A1298C polymorphism and lung cancer risk was found in Caucasian or Asian populations under any genetic models.

Although we performed a subgroup analysis stratified by ethnicity, we failed to perform a subgroup analysis stratified by type of lung cancer or by gender since most of the studies included mixed types of lung cancer in the case group and only a few studies reported the absolute number of homozygous and heterozygous individuals in case and control groups stratified by gender. As we know, the reliability of a meta-analysis based on small sample sizes is not high. Future studies stratified by type of lung cancer or by gender are therefore needed.

Through meta-analysis, a statistical technique for combining the results from independent studies, we were able to draw more reliable conclusions on the influence of the MTHFR C677T and A1298C polymorphisms on lung cancer risk. However, as lung cancer is believed to involve many factors, genetic and/or environmental, future research should investigate not only individual genes, but also gene-gene interactions, genetic-nutritional interactions, and other SNPs.

Several potential limitations of this meta-analysis should be mentioned. Although the funnel plot and the Begg test showed no publication bias, selection bias may have occurred because only studies in English or Chinese were selected. There was also significant heterogeneity detected for the C677T polymorphism. Despite these limitations, our meta-analysis has some clear advantages. Our meta-analysis contains the largest sample size to date to assess the association between the MTHFR C677T and A1298C polymorphisms and lung cancer risk. We were able to perform a subgroup analysis stratified by ethnicity, and the sensitivity analysis showed that no single study strongly affected the combined results. The well-designed search and selection method significantly increased the statistical power of this meta-analysis, and no publication bias was detected, indicating that our pooled results are likely to be reliable.

In summary, our meta-analysis showed that the MTHFR C677T polymorphism was associated with a significantly increased lung cancer risk in Asian and overall populations, but not in Caucasian populations. However, no significant association between the MTHFR A1298C polymorphism and lung cancer risk was found in Caucasian or Asian populations under any genetic models. Future research should investigate not only individual genes, but also gene-gene interactions, genetic-nutritional interactions, and other SNPs.

\section{Conflicts of interest}

The authors declare no conflict of interest.

\section{REFERENCES}

Arslan S, Karadayi S, Yildirim ME, Ozdemir O, et al. (2011). The association between methylene-tetrahydrofolate reductase gene polymorphism and lung cancer risk. Mol. Biol. Rep. 38: 991-996.http://dx.doi.org/10.1007/s11033-010-0194-z

Boccia S, Boffetta P, Brennan P, Ricciardi G, et al. (2009). Meta-analyses of the methylenetetrahydrofolate reductase C677T and A1298C polymorphisms and risk of head and neck and lung cancer. Cancer Lett. 273: 55-61.http://dx.doi. org/10.1016/j.canlet.2008.07.026

Cai ZX, Zu X and Huang FF (2014). Relationship between gene polymorphisms of methylenetetrahydrofolate reductase enzyme and lung cancer in Henan Han population. J. Chin. Pract. Diagn. Ther. 28: 866-868. 
Cavic M, Krivokuca A, Spasic J, Brotto K, et al. (2014). The influence of methylenetetrahydrofolate reductase and thymidylate synthetase gene polymorphisms on lung adenocarcinoma occurrence. J. BUON 19: 1024-1028.

Cheng Z, Wang W and Song YN (2011). Association between C677T genetic polymorphisms of methylenetetrahydrofolate reductase and risk of lung cancer. Chin. J. Tuberc. Respir. Dis. 34: 57-58.

Cheng Z, Wang W, Dai LL and Kang Y (2012). MTHFR C667T polymorphism association with lung cancer risk in Henan province: a case-control study. Asian Pac. J. Cancer Prev. 13: 2491-2494.http://dx.doi.org/10.7314/APJCP.2012.13.6.2491

Cui LH, Shin MH, Kim HN, Song HR, et al. (2011a). Methylenetetrahydrofolate reductase C677T polymorphism in patients with lung cancer in a Korean population. BMC Med. Genet. 12: 28.http://dx.doi.org/10.1186/1471-2350-12-28

Cui LH, Yu Z, Zhang TT, Shin MH, et al. (2011b). Influence of polymorphisms in MTHFR 677 C $\rightarrow$ T, TYMS $3 R \rightarrow 2 R$ and MTR $2756 \mathrm{~A} \rightarrow \mathrm{G}$ on NSCLC risk and response to platinum-based chemotherapy in advanced NSCLC. Pharmacogenomics 12: 797-808.http://dx.doi.org/10.2217/pgs.11.27

Gemignani F, Landi S, Szeszenia-Dabrowska N, Zaridze D, et al. (2007). Development of lung cancer before the age of 50: the role of xenobiotic metabolizing genes. Carcinogenesis 28: 1287-1293.http://dx.doi.org/10.1093/carcin/bgm021

Goyette P, Sumner JS, Milos R, Duncan AM, et al. (1994). Human methylenetetrahydrofolate reductase: isolation of cDNA mapping and mutation identification. Nat. Genet. 7: 551.http://dx.doi.org/10.1038/ng0694-195

Heijmans BT, Boer JM, Suchiman HE, Cornelisse CJ, et al. (2003). A common variant of the methylenetetrahydrofolate reductase gene (1p36) is associated with an increased risk of cancer. Cancer Res. 63: 1249-1253.

Higgins JP and Thompson SG (2002). Quantifying heterogeneity in a meta-analysis. Stat. Med. 21: 1539-1558.http://dx.doi. org/10.1002/sim.1186

Hung RJ, Hashibe M, McKay J, Gaborieau V, et al. (2007). Folate-related genes and the risk of tobacco-related cancers in Central Europe. Carcinogenesis 28: 1334-1340.http://dx.doi.org/10.1093/carcin/bgm067

Jeng YL, Wu MH, Huang HB, Lin WY, et al. (2003). The methylenetetrahydrofolate reductase $677 \mathrm{C} \rightarrow$ T polymorphism and lung cancer risk in a Chinese population. Anticancer Res. 23 (6D): 5149-5152.

Jing C, Zhang YH and Peng MF (2007). Study of the relationship between C677T, A1298C genepolymorphisms of methylenetetrahydrofolate reductase and lung cancer. Chin. Clin. Oncol. 12: 0671-0675. (In Chinese)

Kiyohara C, Horiuchi T, Takayama K and Nakanishi Y (2011). Methylenetetrahydrofolate reductase polymorphisms and interaction with smoking and alcohol consumption in lung cancer risk: a case-control study in a Japanese population. BMC Cancer 11: 459.http://dx.doi.org/10.1186/1471-2407-11-459

Krajinovic M, Lamothe S, Labuda D, Lemieux-Blanchard E, et al. (2004). Role of MTHFR genetic polymorphisms in the susceptibility to childhood acute lymphoblastic leukemia. Blood 103:252-257.http://dx.doi.org/10.1182/blood-2003-06-1794

Liu CS, Tsai CW, Hsia TC, Wang RF, et al. (2009). Interaction of methylenetetrahydrofolate reductase genotype and smoking habit in Taiwanese lung cancer patients. Cancer Genomics Proteomics 6: 325-329.

Liu H, Jin G, Wang H, Wu W, et al. (2008). Association of polymorphisms in one-carbon metabolizing genes and lung cancer risk: a case-control study in Chinese population. Lung Cancer 61: 21-29.http://dx.doi.org/10.1016/j.lungcan.2007.12.001

Ma QL, Li YF and Ji M (2012). Study of the association between C677T gene polymorphisms of methylenetetrahydrofolate reductase and susceptibility to lung cancer. Zhonghua Linchuang Yishi Zazhi 6: 213-215.

Mao R, Fan Y, Jin Y, Bai J, et al. (2008). Methylenetetrahydrofolate reductase gene polymorphisms and lung cancer: a metaanalysis. J. Hum. Genet. 53: 340-348.http://dx.doi.org/10.1007/s10038-008-0262-6

Norton HW and Neel JV (1965). Hardy-Weinberg equilibrium and primitive populations. Am. J. Hum. Genet. 17: 91-92.

Parle-McDermott A, Mills JL, Molloy AM, Carroll N, et al. (2006). The MTHFR 1298CC and 677TT genotypes have opposite associations with red cell folate levels. Mol. Genet. Metab. 88: 290-294.http://dx.doi.org/10.1016/j.ymgme.2006.02.011

Shen H, Spitz MR, Wang LE, Hong WK, et al. (2001). Polymorphisms of methylene-tetrahydrofolate reductase and risk of lung cancer: a case-control study. Cancer Epidemiol. Biomarkers Prev. 10: 397-401.

Shen $\mathrm{H}$, Wei Q, Pillow PC, Amos Cl, et al. (2003). Dietary folate intake and lung cancer risk in former smokers: a case-control analysis. Cancer Epidemiol. Biomarkers Prev. 12: 980-986.

Shen M, Rothman N, Berndt SI, He X, et al. (2005). Polymorphisms in folate metabolic genes and lung cancer risk in Xuan Wei, China. Lung Cancer 49: 299-309.http://dx.doi.org/10.1016/j.lungcan.2005.04.002

Shi Q, Zhang Z, Li G, Pillow PC, et al. (2005). Sex differences in risk of lung cancer associated with methylene-tetrahydrofolate reductase polymorphisms. Cancer Epidemiol. Biomarkers Prev. 14: 1477-1484.http://dx.doi.org/10.1158/1055-9965.EPI$\underline{04-0905}$

Shields PG (2002). Molecular epidemiology of smoking and lung cancer. Oncogene 21: 6870-6876.http://dx.doi.org/10.1038/ sj.onc. 1205832

Siemianowicz K, Gminski J, Garczorz W, Slabiak N, et al. (2003). Methylenetetrahydrofolate reductase gene C677T and A1298C polymorphisms in patients with small cell and non-small cell lung cancer. Oncol. Rep. 10: 1341-1344.

Stroup DF, Berlin JA, Morton SC, Olkin I, et al. (2000). Meta-analysis of observational studies in epidemiology: a proposal for reporting. Meta-analysis Of Observational Studies in Epidemiology (MOOSE) group. JAMA 283: 2008-2012.http://dx.doi. org/10.1001/jama.283.15.2008 
Suzuki T, Matsuo K, Hiraki A, Saito T, et al. (2007). Impact of one-carbon metabolism-related gene polymorphisms on risk of lung cancer in Japan: a case control study. Carcinogenesis 28: 1718-1725.http://dx.doi.org/10.1093/carcin/bgm104

Torre LA, Bray F, Siegel RL, Ferlay J, et al. (2015). Global cancer statistics, 2012. CA Cancer J. Clin. 65: 87-108.http://dx.doi. org/10.3322/caac.21262

van Rens MT, de la Rivière AB, Elbers HR and van Den Bosch JM (2000). Prognostic assessment of 2,361 patients who underwent pulmonary resection for non-small cell lung cancer, stage I, II, and IIIA. Chest 117: 374-379.http://dx.doi. org/10.1378/chest.117.2.374

Voorrips LE, Goldbohm RA, Brants HA, van Poppel GA, et al. (2000). A prospective cohort study on antioxidant and folate intake and male lung cancer risk. Cancer Epidemiol. Biomarkers Prev. 9: 357-365.

Yang XX, Li FX and Yi JP (2010). Association between C677T genetic polymorphisms of methylenetetrahydrofolate reductase and risk of lung cancer, gastric cancer, and colorectal cancer. Guangdong Med. J. 31: 2375-2378.

Yao QF, Chen X and Xue JR (2010). Relationship of polymorphisms of MTHFR gene and hypermethylation of tumor suppressor gene in lung cancers. Cancer Prev. Treat 37: 531-534.

Yilmaz M, Kacan T, Sari I and Kilickap S (2014). Lack of association between the MTHFRC677T polymorphism and lung cancer in a Turkish population. Asian Pac. J. Cancer Prev. 15: 6333-6337.http://dx.doi.org/10.7314/APJCP.2014.15.15.6333

Zhang XM, Miao XP, Tan W, Qu SN, et al. (2005). Association between genetic polymorphisms in methylentetrahydrofolate reductase and risk of lung cancer. Zhongguo Yi Xue Ke Xue Yuan Xue Bao 27: 700-703.

Zou QF, Chen SQ and Ma L (2006). Polymorphisms of 5,10-methylenetetrahydrofolate reductase and risk of lung cancer in Guangdong population. Cancer Res. Prev. Treat. 33: 572-574. 